\title{
An Exploration into Vection During Virtual Helicopter Flight Using Varying Levels of Active Control and Passive Control
}

Lars Kooijman*, Houshyar Asadi, Shehab Abdulraqeb, Wadhah Al-Ashwal, Shady Mohamed, and Saeid Nahavandi.

Institute for Intelligent Systems Research and Innovation (IISRI), Deakin University, Australia

* Corresponding author: Lars Kooijman, email: kooijman.1@outlook.com

\begin{abstract}
The sensation of self-motion, which is called vection, is a phenomenon that is traditionally measured in passive participants. Research on vection during active control is often done through subjective reports using single intensity stimuli. Herein, we investigated how varying difficulty levels of active control as well as passive control affects subjective vection intensity and objective physiological signals. Participants were visually and audibly immersed in a virtual environment wherein either they or an AI controlled a helicopter. Electrodermal activity and accelerations of the body core were recorded during flight. Participants rated the intensity of vection and object-motion for 3 sensory modalities after each trial. Marginally higher vection compared to object-motion ratings were found. Vection ratings were lightly affected by control difficulty due to weather conditions. Our results show that vection can be elicited during active and passive control activities and including objective measures could elevate our understanding of vection.
\end{abstract}




\section{Introduction}

Vection is a phenomenon also known as self-motion perception which occurs daily as we move through the environment. Vection has often been described as a visual illusion of self-motion, however, recently a novel definition has been suggested which encompasses real and illusory vection across multiple sensory modalities [1]. The elicitation of vection is suggested to be an important element to enhance the immersiveness of a virtual environment [2], however, it is also suggested that vection is related to visuallyinduced motion sickness (e.g., see [3] for a review).

The characteristics and features of the visual stimuli directly affect vection intensity. For example, Bubka and Bonato [4] found that the addition of color, as well as bob and sway (i.e., visual field movements due to walking), reduced vection onset and increased vection magnitudes. Evidence on the effect of different visual characteristics on vection can also be found outside of the laboratory. Ungs [5] assessed the occurrence of illusory vection in helicopter pilots and found from the pilot reports that vection was more likely to occur in dark lighting conditions and over rough seas than in light conditions and over smooth seas. Therefore, it appears that naturalistic movement cues, such as visual sway, and environmental conditions, such as wind and light, can enhance the subjective experience of vection.

Besides stimulus content, the activity of the participant also appears to influence vection. Vection is often measured while participants are passive and in a stationary position (e.g., [4], [6], [7]), but a few studies in the literature investigated vection wherein participants were able to move or in control. For example, Clifton and Palmisano [8] found higher vection ratings when participants navigated a virtual environment by steering an avatar compared to when participants displaced themselves via teleporting. A similar effect was found by Seno et al. [9], who reported that passengers experienced less vection compared to drivers. Conversely, Riecke and Feuereissen [10] found that active control reduced vection intensity and increased vection onset latency. Therefore, there is inconclusive evidence whether active control enhances or decreases subjective vection experiences especially in using aircrafts.

Most vection studies rely on the self-reported measures (e.g., vection intensities or magnitude estimations) to assess vection and investigate only one intensity or difficulty level of active control. Although electroencephalograms have previously been used as an objective measure in vection research (see [11] for a review), the majority of vection studies do not record physiological signals concurrently to subjective measures corroborate their findings. To the best of our knowledge, the concurrent measurement of physiological signals to vection intensity ratings for an active control task has not been done.

Herein, we aim to identify how vection is affected by active and passive control and how vection intensity is modulated by varying difficulty levels in active control operationalized through different weather conditions. We hypothesized that adverse weather conditions (e.g., strong winds and rain) would result in a more intense experience of vection compared to more favorable weather conditions due to increased roll of the helicopter and the presence of rain particles suggesting forward motion. Furthermore, an increase in electrodermal activity and absolute accelerations of the body were expected for conditions with higher selfreported vection intensities. Lastly, we hypothesize that active control would increase vection intensity ratings compared to passive control conditions as participants' will be more immersed by focusing on the

control of the aircraft and the associated motion cues. We chose to use helicopter flight as it would be more challenging than driving a land-based vehicle. 


\section{Methods}

\section{Participants}

Twelve participants (11 males, 1 did not disclose their gender) were recruited from the student and staff body of Deakin University. 3 participants were aged between 18 and 24, and 9 participants were aged between 25 and 34. All participants provided written informed consent at the start of the experiment. This study was approved by Deakin University's Human Ethics Advisory Group (HEAG).

\section{Materials}

Participants controlled the virtual helicopter in Xplane v11.52 64-bit software via the HeliMod. The HeliMod is a stationary helicopter simulation control platform that allows users to operate a virtual helicopter using cyclic, collective, and anti-torque pedal control [12]. The Helimod was connected via USB to a desktop computer running a 64-bit version of Windows 10 with Intel(R) Core(TM) i7-9700 CPU @ 3.60GHz, NVIDIA GeForce RTX 2080 Super 8GB graphics card, Z390 X-CF MOBO and 32GB RAM. The desktop also ran a custom .NET protocol that extracted data from Xplane and ran SteamVR software to enable the use of an HTC Vive Head-Mounted Display (HMD). The HMD was used to visually and audibly immerse participants in the virtual environment. Electrodermal activity (EDA) and accelerations of the body core (Acc) were recorded using the Equivital EQ-02. For the familiarization session, a 65" Bauhn ATVU65-0916 television running at 60Hz was used instead of the HMD. The set-up is depicted in Fig. 1.

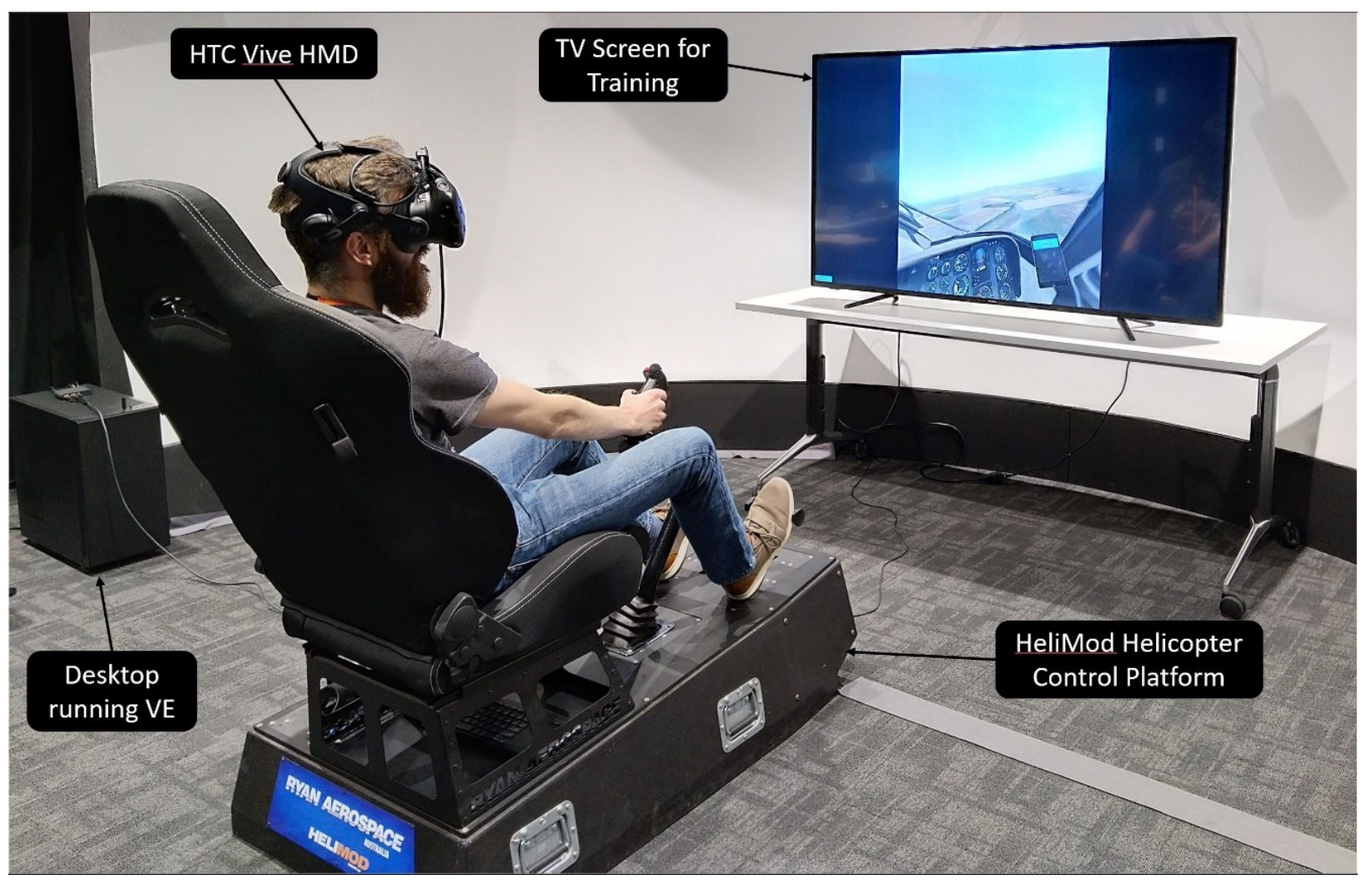

Figure 1: The setup used in the experiment. The participant is seated in the HeliMod, shown wearing the HTC Vive HMD. The projection inside the HMD is shown in duplicate on the TV screen that was used for training purposes. The desktop running the Virtual Environment (VE) is shown in the background 


\section{Design}

The experiment consisted of 2 familiarization sessions of 5 minutes each and 4 flight conditions of 7 minutes each. The first familiarization session involved flying the virtual helicopter freely, controlling it using the HeliMod and viewing the virtual environment from the perspective of the cockpit via the TV screen to allow participants to accustom themselves to the helicopter dynamics first before immersing them in VR and potentially inducing motion sickness. In the second familiarization session participants were again free to fly the helicopter anywhere but were immersed in the virtual environment by means of the HMD instead of viewing it on the TV. Participants viewed the environment from the first-person perspective of the pilot seated in the cockpit of the helicopter.

In three of the four flight conditions (i.e., C1-C3), participants controlled the helicopter and flew it along a pre-instructed path. In one condition (i.e., C4), an AI controlled the helicopter and flew without performing any large turns. The default clear weather settings were used for $\mathrm{C} 1$ and $\mathrm{C} 4$, however, for the other conditions specific weather conditions were programmed as shown in Table 1. The four flight conditions were presented in a randomized order for each participant to control for carryover effects.

Table 1: Weather conditions programmed in Xplane

\begin{tabular}{lllllll}
\hline Condition & Name & Type & Angle of Attack (deg) & Speed (kts) & Gusts (kts) & Altitude (ft) \\
\hline C1 & Clear & Wind & N/A & N/A & N/A & N/A \\
C2 & Broken & Wind & 52 & 7 & 8 & 25000 \\
C2 & Broken & Wind 70 & 7 & 8 & 2500 \\
C3 & Stormy & Wind & 52 & 14 & 16 & 25000 \\
C3 & Stormy & Wind 70 & 14 & 16 & 2500 \\
C3 & Stormy & Rain & & & \\
C4 & AI Flight & Wind & N/A & N/A & N/A & N/A \\
\hline
\end{tabular}

\section{Procedure}

Participants completed a COVID-19 screening form, were instructed about the goal of the experiment and, after signing the consent form, completed a pre-experiment questionnaire. Participants were informed about the function of each of the sensors while they were attached to the participant by one of the experimenters conform the COVID-19 restrictions at that time. Subsequently, participants were instructed on the workings of the HeliMod and performed the two familiarization sessions while obtaining feedback from an experimenter on controlling the helicopter. After the familiarization sessions, an experimenter explained the flying route for the experimental conditions by means of a printed map. Participants were instructed to fly a figure of eight and the points of turning were made identifiable by significant landmarks in the virtual environment. Participants were instructed that if they experienced discomfort due to cyber or motion sickness, they were free to stop the experiment or the experimental condition at any point. During the conditions, an experimenter would ask the participants "How are you feeling?" every minute and participants were free to reply to this. After completing each condition, participants were able to take off the HMD and complete a questionnaire assessing their motion sickness and motion perception during the condition. After all conditions were completed, a final evaluating questionnaire was administered and upon completion the sensors were removed.

\section{Data processing and analysis}

Helicopter parameters recorded from Xplane and physiological data recorded via the Equivital were read in a custom Matlab script and synchronized. All data were linearly interpolated to achieve a constant sampling frequency. Subsequently, data were filtered using a third-order low-pass of bandpass Butterworth filter. Helicopter data were low pass filtered with a cut-off frequency of $1 \mathrm{~Hz}$, whilst accelerometer data 
were band-pass filtered between 0.2 and $5 \mathrm{~Hz}$. EDA was processed by means of a convex optimization approach using the default settings of cvxEDA [13] to separate the phasic component of the EDA from the tonic component.

For each condition and participant, the aircraft and joystick activity were derived by taking the derivative of the roll, pitch and yaw angles and rectifying them. Furthermore, from the data the following objective dependent variables were computed per condition:

- Mean Skin Conductance Response (mSCR in $\mu \mathrm{S})$ : The mean of the phasic component of EDA during the experimental condition.

$$
m S C R=\overline{E D A}_{\text {phasic, } \text { Flight }}
$$

- The absolute average acceleration during each condition (Acc in $\mathrm{mG}$ ): The absolute average of the three axes of acceleration of the body during each of the experimental conditions.

$$
A c c=\frac{\left(\left|A c c_{X}\right|+\left|A c c_{Y}\right|+\left|A c c_{Z}\right|\right)}{3}
$$

The questionnaire that was completed after each condition contained six statements, denoted in Table 2, that participants rated using an 11-point scale. The six items aimed to investigate participants' vection and object-motion perception via three sensory modalities during the experimental condition. The value 0 represented "None" and the value 10 represented "Severe".

As our sample size is rather small and the experiment is of exploratory nature, we will refrain from using p-values to interpret our results and instead evaluate the data using point estimates, similar to [14]. We thereby adhere to recent calls in the scientific field to abandon the p-value as an indicator of significant or nonsignificant effects (e.g., [15]). Nonetheless, to help readers interpret the differences between values we depict and report within-subject compatibility intervals (i.e., substitute for $95 \%$ confidence intervals, see [15]) per variable by subtracting the mean score of all conditions per participant [16].

Table 2: Six questions related to motion perception

\begin{tabular}{llll}
\hline Question Number & $\begin{array}{l}\text { Motion } \\
\text { Perception }\end{array}$ & $\begin{array}{l}\text { Sensory } \\
\text { Modality }\end{array}$ & Questionnaire Item $^{\mathrm{a}}$ \\
& &
\end{tabular}

\begin{tabular}{llll}
\hline 1 & Vection & Visual & I saw I was moving/rotating \\
2 & Vection & Auditory & I heard I was moving/rotating \\
3 & Vection & Vestibular & I felt I was moving/rotating \\
4 & Object-Motion & Visual & I saw the environment was moving/rotating \\
5 & Object-Motion & Auditory & I heard the environment was moving/rotating \\
6 & Object-Motion & Vestibular & I felt the environment was moving/rotating \\
\hline
\end{tabular}

a Items were rated on an 11-point scale with the left anchor with the value 0 stating "None" and the right anchor with the value 10 stating "severe".

\section{Results}

All participants completed the experiment, however, one participant aborted the stormy weather condition (i.e., C3) before the 7 minutes had passed due to a negative experience related to motion sickness and one participant aborted the AI flight condition (i.e., C4) for similar reason. A total of 8 crashes occurred over a total of 48 trials. 


\section{Aircraft and Participant Activity}

Figure 2 shows the activity of the aircraft (left) and participants' joystick (right). The aircraft exhibited considerably more roll activity during the stormy weather condition compared to the broken and clear weather condition as can be seen by the non-overlapping compatibility intervals. Pitch activity was, on average, higher during the stormy weather condition compared to the broken and clear weather condition but was only considerably different from the clear weather condition, as indicated by the nonoverlapping confidence intervals. Moreover, aircraft activity was considerably higher during the active conditions compared to the AI Flight condition.
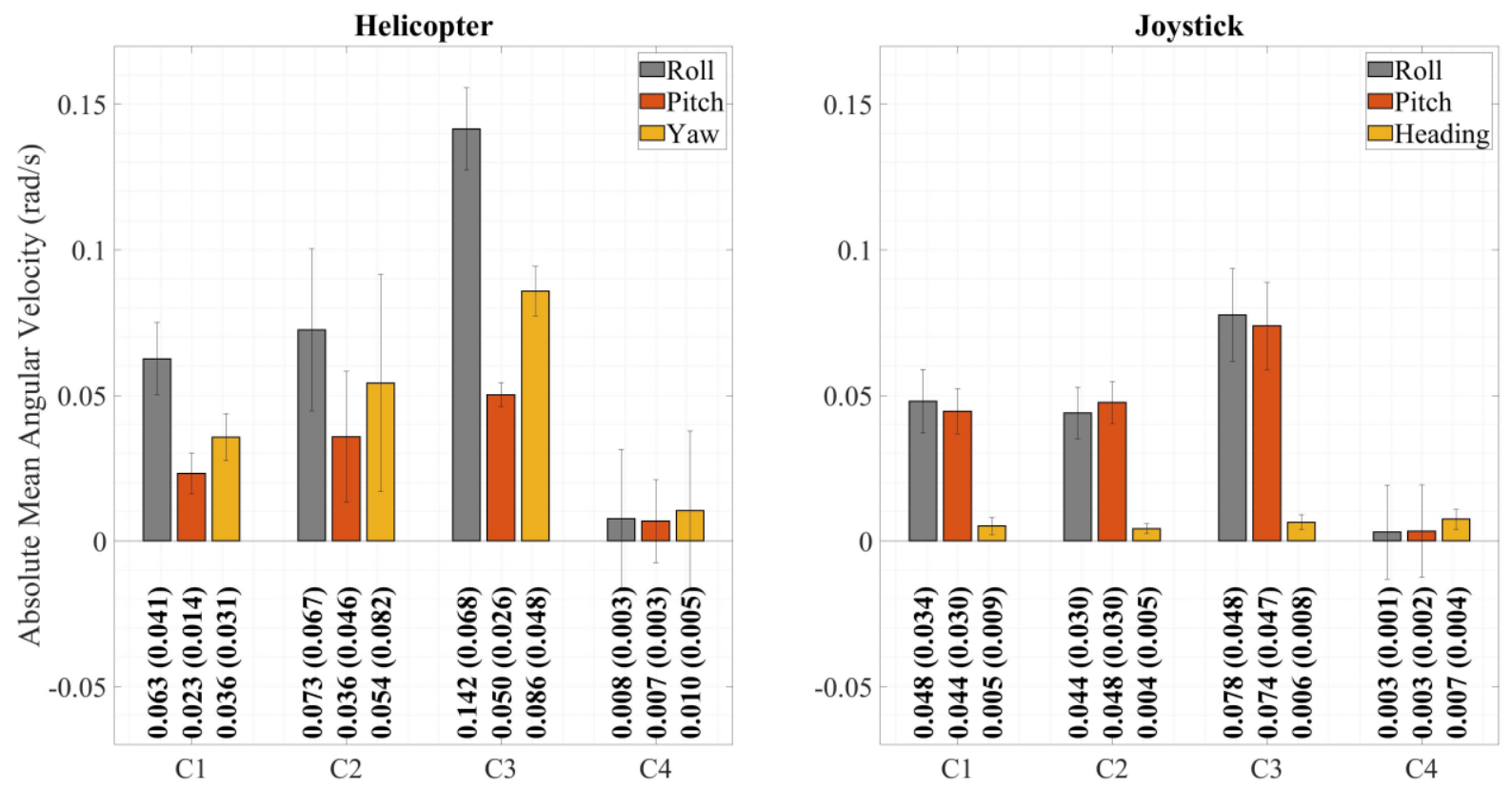

Figure 2: Aircraft and joystick activity for each experimental condition. Normalised means, with standard deviations in brackets, are reported below each bar. Errorbars represent the compatibility interval. $\mathrm{C} 1=$ Clear weather condition, $\mathrm{C} 2=$ broken weather condition, $\mathrm{C} 3=$ Stormy weather condition, $\mathrm{C} 4=\mathrm{AI}$ flight condition .

Similarly, participants' pitch and roll joystick activity was considerably higher during the stormy weather condition compared to the clear and broken weather condition as indicated by the nonoverlapping confidence intervals, however, yaw activity did not differ between conditions. Moreover, it can also be seen that joystick activity was virtually absent during the AI flight conditions.

\section{Physiological Data}

Table 3 shows the mean SCR and the absolute mean accelerations of the body core per experimental condition separately. On average, participants exhibited the lowest $\mathrm{mSCR}$ in the clear weather condition, however, the electrodermal activity of all participants grouped did not differ considerably between conditions as can be seen from the overlapping compatibility intervals.

Table 3: Physiological Data. Means (SD) and compatibility intervals of participants' electrodermal activity and accelerations of the body core for the four different experimental conditions.

\begin{tabular}{llll}
\hline Condition & Variable & Mean (SD) & Compatibility Interval \\
\hline Clear & $\operatorname{mSCR}^{\mathrm{a}}(\mu S)$ & $1.46(1.83)$ & {$[0.122 .80]$} \\
Broken & $\operatorname{mSCR}(\mu S)$ & $2.72(6.61)$ & {$[-2.13,7.57]$} \\
Stormy & $\operatorname{mSCR}(\mu S)$ & $2.05(2.57)$ & {$[0.16,3.93]$} \\
AI Flight & $\operatorname{mSCR}(\mu S)$ & $3.14(5.72)$ & {$[-1.06,7.33]$}
\end{tabular}




\begin{tabular}{llll}
\hline Condition & Variable & Mean $(\mathrm{SD})$ & Compatibility Interval \\
\hline Clear & & & \\
Broken & Acc $^{\mathrm{b}}(\mathrm{mG})$ & $2.16(1.30)$ & {$[1.21,3.12]$} \\
Stormy & Acc $(\mathrm{mG})$ & $2.03(0.93)$ & {$[1.34,2.71]$} \\
AI Flight & Acc $(\mathrm{mG})$ & $2.65(1.20)$ & {$[1.77,3.53]$} \\
\hline
\end{tabular}

${ }^{\mathrm{a}} \mathrm{mSCR}=$ Mean skin conductance response. ${ }^{\mathrm{b}}$ Acc $=$ Average accelerations of the body core.

Accelerations of the body core were, on average, larger during the stormy weather condition compared to the broken and clear weather condition as well as the AI condition. However, the overlapping compatibility intervals indicate that the differences in body core activity between conditions were not substantial.

\section{Questionnaire Responses}

Figure 3 shows participants' mean responses to each of the questions for each condition. Participants reported, on average, higher visual vection for every condition compared to auditory and vestibular. The questions related to vection perception (i.e., Q1-3) were, on average, rated higher than the questions related to object-motion (i.e., Q4-6). However, object-motion ratings did not differ substantially between conditions nor between sensory modality as can be seen by the overlapping compatibility intervals.

For every condition, visual and auditory vection perception was rated considerably more severe compared to visual and auditory object-motion perception, as indicated by the nonoverlapping compatibility intervals. However, the responses per sensory modality and motion perception type did not differ considerably between conditions, as can be seen by the overlapping compatibility intervals.

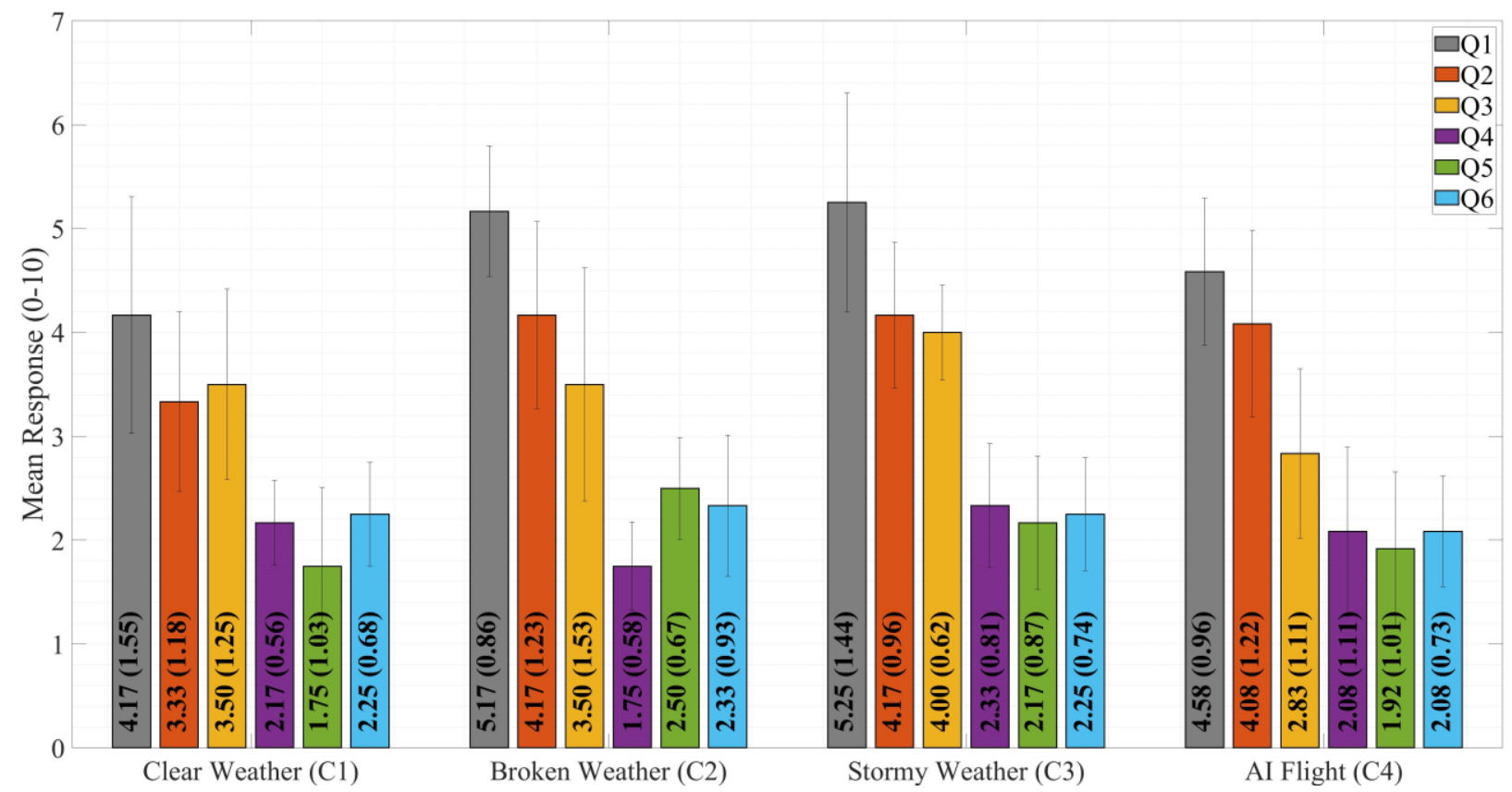

Figure 3: Mean responses of participants to each question for each experimental condition. Normalised means, with standard deviations in brackets, are reported within each bar. Errorbars represent the compatibility interval. Q1 = I saw I was moving/rotating. Q2 = I heard I was moving/rotating. Q3 = I felt I was moving/rotating. Q4 = I saw the environment was moving/rotating. Q5 = I heard the environment was moving/rotating. Q6 = I felt the environment was moving/rotating. 


\section{Discussion}

Our experiment aimed to identify how varying difficulty levels of active control as well as passive control of a virtual aircraft affected subjective vection intensity, electrodermal activity and accelerations of the body core. Participants were visually and audibly immersed in a virtual environment wherein they either actively or passively flew a helicopter. We observed that adverse weather conditions increased the roll activity of the aircraft as well as participants' joystick activity, indicating that the presence of strong winds required the participant to increase their efforts in stabilizing the aircraft. We found that subjective vection ratings were marginally higher during stormy weather conditions compared to clear weather conditions, but the overlap of both the upper and lower compatibility intervals between the experimental conditions for each of the self-motion ratings indicate that no important effects of weather condition were compatible with self-reported vection. Additionally, visual and auditory vection intensity ratings were, on average, higher during the AI Flight condition compared to the clear weather condition. As these two conditions had similar environmental characteristics, this suggests the potential of active control to decrease the intensity of vection compared to passive conditions. However, the overlapping compatibility intervals indicate that the difference in control type were not compatible as an effect on vection intensity. Higher average self-reported ratings of vection intensity compared to the ratings of object-motion were present for all sensory modalities. Vection ratings were, on average, the highest for visual and auditory vection compared to vestibular vection, which matches the modalities we stimulated in this experiment.

Vestibular vection ratings were the lowest in the AI flying condition. The compatibility interval of the vestibular vection in this condition overlaps more with the object-motion ratings compared to the other vection ratings, which could, in part, be explained by a lack of control during the AI flying condition. The higher self-reported vection measures during active control conditions compared to the passive condition could be caused by a heuristic approach when participants assessed their vection during the virtual experience; participants could have used the immersiveness of the experience as a proxy while rating their experience of vection. This heuristic could also explain the marginal differences in self-reported vection intensities between conditions. The adverse weather conditions caused more perturbations on the helicopter and required the participant to increase their efforts to stabilize the aircraft as inferred from the joystick activity and the accelerations of the participants' body. Although accelerations of the body core during the stormy weather conditions were, on average, higher compared to the clear weather condition, the overlap of both the upper and lower compatibility intervals indicate that no important effects of weather condition were compatible with the activity of participants' body core.

The mean skin conductance responses (mSCR) were, on average, higher during the stormy weather condition compared to the clear weather condition suggesting an effect of weather condition on electrodermal activity. However, a few substantially large SCRs were present which could have confounded the dataset due to the low number of participants. Moreover, the feedback of the participants at each minute of enquiry during the conditions comprised mostly of the absence or presence of motion sickness related symptoms and therefore did not provide us with any insights regarding the perception of vection. Therefore, the higher skin conductance responses in stormy weather compared to clear weather could, in part, be explained by either arousal (e.g., see [17] for the effect of arousal on EDA) or motion sickness (e.g., see [18] for the effect of motion sickness on EDA) due to the difficulty in controlling the aircraft.

This study has a few limitations, primarily our use of academic student/staff sample who do not have flight experience. Secondly, it has been shown that task instructions can affect vection experiences [19]. We used a fixed-based simulator which could have instilled a negative cognitive bias in participants towards the occurrence of vection. 
It is concluded that vection can be elicited during active and passive control tasks. The marginal increase in vection intensity rating during adverse compared to favorable weather conditions may be due to the environmental characteristics, such as strong winds and the presence of rain, or due to an increase in control difficulty, however, further research is needed. Furthermore, our findings indicate that the measurement of physiological signals can assist in interpreting the subjective reports of participants. In future experiments, we recommend implementing a temporal measure of vection (e.g., a button press) concurrent to the active control task to ensure the onset and duration of vection is captured and can be correlated to the changes in physiological signals. Furthermore, the addition of motion cues through a motion platform could be interesting to add as it might affect participants' reports on vestibular vection.

\section{ACKNOWLEDGMENTS}

This research was supported by the Australian Research Council (ARC) (Project ID: DE210101623) as well as by the Institute of Intelligent Systems Research and Innovation. The authors would like to thank Ahmad Abu Alqumsan for his help on explaining the use of the sensors and Darius Nahavandi for the discussion in the early stage of the project. The authors would like to acknowledge that this research was conducted on the land of the Wadawurrung people of the Kulin Nation, the traditional owners of the lands, and we would like to pay our respect to their Elders past and present and emerging.

\section{REFERENCES}

[1] S. Palmisano, R. S. Allison, M. M. Schira, and R. J. Barry, "Future challenges for vection research: definitions, functional significance, measures, and neural bases," Front. Psychol., vol. 6, p. 193, 2015, doi: 10.3389/fpsyg.2015.00193.

[2] L. Hettinger, T. N. Schmidt-Daly, D. L. Jones, and B. Keshavarz, "Illusory Self-Motion in Virtual Environments," 2014.

[3] B. Keshavarz, B. E. Riecke, L. J. Hettinger, and J. L. Campos, "Vection and visually induced motion sickness: how are they related?," Front. Psychol., vol. 6, p. 472, 2015, doi: 10.3389/fpsyg.2015.00472.

[4] A. Bubka and F. Bonato, "Natural visual-field features enhance vection.," Perception, vol. 39, no. 5, pp. 627635, 2010, doi: 10.1068/p6315.

[5] T. J. Ungs, "The occurrence of the vection illusion among helicopter pilots while flying over water.," Aviat. Space. Environ. Med., vol. 60, no. 11, pp. 1099-1101, Nov. 1989.

[6] B. Keshavarz, M. Speck, B. Haycock, and S. Berti, "Effect of different display types on vection and its interaction with motion direction and field dependence," Iperception., vol. 8, no. 3, p. 2041669517707768 , 2017, doi: 10.1177/2041669517707768.

[7] F. Soave, N. Bryan-Kinns, and I. Farkhatdinov, "A Preliminary Study on Full-Body Haptic Stimulation on Modulating Self-motion Perception in Virtual Reality," in Augmented Reality, Virtual Reality, and Computer Graphics, 2020, pp. 461-469.

[8] J. Clifton and S. Palmisano, "Effects of steering locomotion and teleporting on cybersickness and presence in HMD-based virtual reality," Virtual Real., vol. 24, no. 3, pp. 453-468, 2020, doi: 10.1007/s10055-019-004078.

[9] T. Seno, Y. Fujii, and T. Yoshinaga, "Active Control of the Direction of Self-Motion by Head Movements and Vection Strength-The Comparison between the Virtual Driver and Virtual Passenger-," Vision, vol. 30, no. 2, pp. 65-71, 2018, doi: 10.24636/vision.30.2_65.

[10] B. E. Riecke and D. Feuereissen, "To Move or Not to Move: Can Active Control and User-Driven Motion Cueing Enhance Self-Motion Perception ('vection') in Virtual Reality?," in Proceedings of the ACM Symposium on Applied Perception, 2012, pp. 17-24, doi: 10.1145/2338676.2338680.

[11] S. Berti and B. Keshavarz, "Neuropsychological Approaches to Visually-Induced Vection: an Overview and Evaluation of Neuroimaging and Neurophysiological Studies.," Multisens. Res., vol. 34, no. 2, pp. 153-186, Aug. 2020, doi: 10.1163/22134808-bja10035.

[12] Ryanearospace.com, "Helimod Mark III," 2021. https://www.ryanaerospace.com.au/HELIMOD-Mark-III.

[13] A. Greco, G. Valenza, A. Lanata, E. P. Scilingo, and L. Citi, "cvxEDA: A Convex Optimization Approach to Electrodermal Activity Processing," IEEE Trans. Biomed. Eng., vol. 63, no. 4, pp. 797-804, 2016, doi: 10.1109/TBME.2015.2474131. 
[14] Y. B. Eisma, A. Reiff, L. Kooijman, D. Dodou, and J. C. F. de Winter, "External human-machine interfaces: Effects of message perspective," Transp. Res. Part F Traffic Psychol. Behav., vol. 78, pp. 30-41, 2021, doi: https://doi.org/10.1016/j.trf.2021.01.013.

[15] V. Amrhein, S. Greenland, and B. McShane, "Scientists rise up against statistical significance.," Nature, vol. 567, no. 7748, pp. 305-307, Mar. 2019, doi: 10.1038/d41586-019-00857-9.

[16] R. Morey, "Confidence Intervals from Normalized Data: A correction to Cousineau (2005)," 2008.

[17] C.-A. Wang, T. Baird, J. Huang, J. D. Coutinho, D. C. Brien, and D. P. Munoz, "Arousal Effects on Pupil Size, Heart Rate, and Skin Conductance in an Emotional Face Task," Front. Neurol., vol. 9, p. 1029, 2018, doi: $10.3389 /$ fneur.2018.01029.

[18] T. Irmak, D. M. Pool, and R. Happee, "Objective and subjective responses to motion sickness: the group and the individual," Exp. Brain Res., vol. 239, no. 2, pp. 515-531, 2021, doi: 10.1007/s00221-020-05986-6.

[19] S. Palmisano and A. Y. C. Chan, "Jitter and size effects on vection are immune to experimental instructions and demands.," Perception, vol. 33, no. 8, pp. 987-1000, 2004, doi: 10.1068/p5242. 